Informal Report

$i+i$
UC-34b

Reporting Date: October 1975 Issued: November 1975

\title{
A General Numerical Fluid Dynamics Algorithm for Astrophysical Applications
}

by

\author{
H. M. Ruppel \\ L. D. Cloutman
}

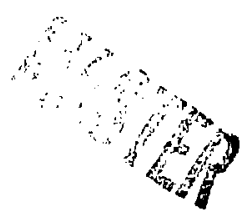


In the interest of prompt distribution, this report was not edited by the Technical Information staff.

Printed in the United Staten of America. Available from National Tochnical Information Service

US Department of Commerce

5285 Por Royal Road

Springfiold VA 2215

Price: Printed Copy $\$$.00 Microfiche $\$ 2.25$

bv The United Hiates Goveramens. Neithor the United States

nor thr LInited States Enerky Research and Development Ad-

minintration, nor any of their employees, mor any of their con.

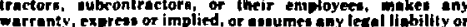

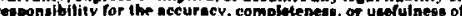

anvinformation. apporatus, product

repreernts that ite use would not infrinter privachly ownet 
This tepout - Notice

This ieport was prepued as in account of wurk

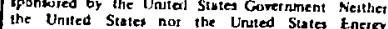

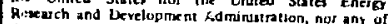

thes einpluyees, nur any of theis contraciots

subcaninctors. or thes employeer, mukes any

witranly, express of Inpted, of elsumes iny Legal

thibdily os responsubuty fos the excuracy, completeness ol uxeluthes of any inf,irmation. apparal us. product or

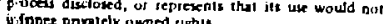

A GENERAL NUMERICAL FLUID DYNAMICS ALGORITHM

FOR ASTROPHYSICAL APPLICATIONS

by

H. M. Ruppel and L. D. Cloutman

ABSTRACT

Finite difference simulation of fluid flows under astrophysical conditions is often complicated by factors such as complex gas physics, the occurrence of dynamics at widely differing length ccales, and the necessity of using implicit difference equations. This report describes a simple, general, and efficient ilgorithm for solving one-dimensional, spherically symmetri: prob? ems using a variation of the ICED-ALE method. A comjuter code named VEGA has been written based on this algorithm, and the early ilages of the collapse of a onesolar mass protostellar cloud are presented as a samrle solution.

\section{INTRODUCTION}

The application of the equations of fluid dynamics to astrophysical problems opers the possibility of studying complex dynamical phases of stellar evolution in considerable detail. However, the numerical algorithms in the astronomical literature tend to have restrictive stability 7 imits; rezoning, when done at al], is done in a primitive, ad hoc fashion. In an attempt to overcome these difficulties we have implemented a variaticn on the YAQU] code of Hirt, Amsden, and Cook, ' he report here the basic outline of our method and the progress we have made in applying it to astrophysics.

In our code, VEGA, the conservation equations are solved in two steps: In phase I the solution is obtained in the coordinate system moving with the fluid; this is called the Lagrangian phase. in Phase II, the rezone or convective phase, the convection terms are added. By rezoning we mean the movement of mesh vertices to maintain a reasonable grid spacing. This is accomplished by defining a grid velocity as some arbitrary fraction of the fluid velocity. This fraction can be a function of space and time. In the limit that the grid velocity is zero the calculation is Eulerian. If the grid velocity is set equal to the fluid velocity, the calculation is Lagrangian. This technique removes the ad hoc nature and numerical difficulties of rezoning by procedures such as the insertifin and deletion of grid points.

Attempts to obtain numerical jolstions to probleirs in scellar evolution must copt: with limits on the time step imposed by the wave speeds associated with the problem. In an explicit cillculation one simply updates the flow variables for each cell in terms of quantities available at the begirning of the calculational cycle. This approach inposes stability requirements that no waves (scund waves, elastic waves, thermal waves, etc.) can travel more than one cell per cycle. This is the well-known Courant condition, which is usually expressed as $c$ it $/ \delta x<1$. where $c$ is the wave speed. To remove this restriction, we employ an implicit scheme that inc: ides in the solution of the equations as much advanced time information as possible. This requires either an iterative scheme to reach a consistent set of advanced time values or a direct solution technique applied to a linearized set of difference equations. 
In the former approach information tends to travel only one cell per iteration, although schemes such as successive overrelaxation (SOR) do prapagate signals more rapidly in one direction if they use updated information as it becomes available. Direct solution techniques couple all nodes simultaneously. In either case, information can travel over many cells in any one cycle, even over the entire mesh if the wave speed is sufficiently great. This allows for much larger time steps since the Courant condition no longer constrains it.

There is a certain amount of controversy associated with implicit schemes. This contraversy originates in the belief that accuracy is lost in the large time steps an implicit approach permits. There is, of course, validity in such a view. However, in many calculations the solution is changing extremely slowly during a portion of the calculation and nothing is lost by a large time step. If the fluid motion is experiencing changes that are of short duration compared to the time $s t \in p$ allowed, the time step must be limited. Otherwise important or interesting phenomena may be unresolved. As an example of the restriction that can be imposed by the wave speed, the largest time steps used in the protostar calculation are sixteen orders of magnitude larger than the explicit thermal diffusion stability limit. We have no reason to believe we have suppressed any short-lived transients that would affect the solution. There are other phenomena associated with the calculation of stellar evolution that pose similar, if less dramatic, problems when treated explicitly. With this in mind we elected to solve the equations describing stellar evolution with as much advanced time information as possible, while making the difference equations tractable. Since the number of cells and the number of variables is significantly smaller in ane dimension than in two or three dimensions, it is feasible to consider a direct solution technique for one-dimensional codes. This requires linearizing the system of equations in the time advanced variables and leads to a banded matrix. The width of the band will depend on the number of dependent variables included implicitly. An important feature of this appreach is the absence of iterations, such as one finds in the standard Henyey technique. The result is an efficient, stable algorithm free from convergence difficulties.

One final consideration which entered into our choice of techniques was the desire to be able to extend the set of equations, increasing the amount of physics to the included, with minimal structural alteration to the code. That is, we wished to be able to add equations, add variables, or change the functional form of constitutive relations, all in a direct and straightforward fashion.

To summarize, three factors played major roles in motivating us to adopt the present approach: we wished to be able to vary the resolution of the calculational mesh and to include a continuous rezone; we wanted to include the gas physics as implicitly as possible to maximize the efficiency of the code; we needed to have the flexibility of altering or adding equations with minimum difficulty. These considerations led us to construct a code divided into two phases: an implicit Lagrangian phase in which we solve the complete linear system for the flow varibles and a rezone phase in which the contributions from the convection tenms are added to the Lagrangian components.

\section{I. EQUATIONS}

As rophysical hydrodynamical algorithms frequently use transformations of the variables in an attempt to improve accuracy. The variable mesh, continuous rezone feature allows us to resolve the solution of most problems with a reasonable number of cells, obviating the need to transform the independent variable. When reasonable resolution is achieved, a transformation of dependent variables is not desirable. Our experience has shown that the use of the primitive variables will give accuracy comparable to any tranjformed variables, and transformed variables almost always lead to more complex, less efficient programs. For a general method such as ours, variable transformations are not worth the trouble.

Let us now examine the set of equations currently in the code, their significance and the details of the solution algorithm. The full mass equation, including a term for turbulent diffusion 2,3 is written as

$\frac{\partial \rho}{\partial t}+\frac{1}{r^{2}} \frac{\partial}{\partial r}\left(r^{2} \rho u\right)=\frac{1}{r^{2}} \frac{\partial}{\partial r}\left(\sigma r^{2} \frac{\partial \rho}{\partial r}\right)$. 
Because we are including the nuclear burning of hydrogen, we need a species equation that will allow for hydrogen to be depleted by conversion to helium.

$\frac{\partial \rho_{H}}{\partial t}+\frac{1}{r^{2}} \frac{\partial}{\partial r}\left(r^{2} \rho_{H} u\right)=\frac{3}{r^{2}} \frac{\partial}{\partial r}\left(\sigma r^{2} \frac{\partial \rho_{H}}{\partial r}\right)+\left(\frac{\delta \rho_{H}}{\delta t}\right)_{n u c}$,

where $\left(s_{\rho_{H}} / \delta t\right)_{\text {nuc }}$ is the nuclear creation rate. The momentum equation contains both a gravitational acceleration, $g$, and a viscous iem, $\Lambda$,

$\frac{3, u}{\partial t}+\frac{1}{r^{2}} \frac{\partial}{\partial r}\left(r^{2} p u^{2}\right)=-p g-\frac{\partial p}{\partial r}+A$,

where

$\Lambda=\frac{1}{r^{2}} \frac{\dot{\partial}}{\partial r}\left(r^{2}(2 \mu+\lambda) \frac{\partial u}{\partial r}\right)-\frac{2 u(2 \mu+y)}{r^{2}}+\frac{2 u}{r} \frac{\dot{\partial}}{\partial r}$,

and

$g=\frac{G}{r^{2}} \int_{0}^{r} 4 \pi \rho x^{2} d x$

For the specific internal energy, I, we write

$$
\begin{aligned}
& \frac{\partial \rho I}{\partial t}+\frac{1}{r^{2}} \frac{\partial}{\partial r}\left(r^{2} \rho u I\right)=-p \frac{1}{r^{2}} \frac{\partial}{\partial r}\left(r^{2} u\right)+\rho \varepsilon \\
& +\frac{1}{r^{2}} \frac{\partial}{\partial r}\left(r^{2} K \frac{\partial T}{\partial r}\right)+2 u\left[\left(\frac{\partial u}{\partial r}\right)^{2}+\frac{2 u^{2}}{r^{2}}\right] \\
& +\lambda\left[\frac{1}{r^{2}} \frac{\partial}{\partial r}\left(r^{2} u\right)\right]^{2}
\end{aligned}
$$

In this equation $E$ is the rate of energy release from nuclear burning and $K$ is the themal conductivity. The temperature is obtained from the specific internal energy through the relation $T=I / c_{v}$, where $c_{v}$ is the specific heat at constant volume. The nuclear energy production rate $\varepsilon$ is discussed more fully in Sec. III. In addition to the conservation equations we include an equation of state of the form
$P=\Gamma\left(\rho, P_{j}, I\right) \Gamma I+\frac{1}{3} a T^{4}$,

where $\Gamma$ is a function which includes the real gas effects such as variable mean molecular weight and electron degeneracy. For a simpie gamma-law ideal gas neglecting radiation pressure, $\Gamma$ is merely $(y-1)$. More complicated cases are discussed in the Appendix.

As an example of our solution technique, consider the equation for the internal energy in the form

$\frac{\partial \rho I}{\partial t}+\nabla \cdot(\partial I \vec{u})=R$,

where $R$ includes the work, diffusion, and source terms. Paralleling the approach of Hirt et al., we integrate this equation over a control volume $V$, which for a cell-centered quantity, like $\rho I$, is simply a calculational cell. We obtain in this way

$\iiint_{V} \frac{\partial}{\partial t}(\rho I) d^{3} V+\iiint_{V} \nabla \cdot(\rho I \vec{u}) d^{3} V=\iiint_{V} R d^{3} V$.

We also make use of the we!l-known relation 4

$\frac{d}{d t} \iiint_{V} \rho I d^{3} v=\iiint_{V} \frac{\partial}{\partial t}(\rho I) d^{3} v+\iint_{S} \rho l \vec{u}_{G} \cdot \hat{n} d^{2} S$.

This relation states that the total change of a quantity in a control volume is made up of two parts: the change with time within the volume and the amount taken into the volume as the surface, $S$, of the control volume moves with velocity $\vec{u}_{g}$. We will refer to this velocity as the grid velocity. For a Lagrangian calculation, $\vec{u}_{g}=\vec{u}$. For an Eulerian calculation, $\overrightarrow{\mathrm{u}}_{\mathrm{g}}=0$.

Combining Eqs. (9) and (10) and using the divergence theorem yields

$\frac{d}{d t} \iiint_{V} \rho I d^{3} V-\iint_{S} \rho I\left(\vec{u}_{g}-\vec{u}\right) \cdot \hat{n} d^{2} S=\iiint_{V} R d^{3} V$. 
Although we have examined only the equation for $\rho I$ in detail, each of the conservation equations will contain an integral of the form

$$
\iint_{S} \rho Q\left(\vec{u}_{g}-\vec{u}\right) \cdot R d S \text {. }
$$

As we mentioned above, in a Lagrangian calculation $\vec{u}_{g}=\vec{u}$, and this term vanishes. It is this structure that leads one to solve the equations without this term first and then to add its contribution as a separate step in the rezone or convective phase. We should comment that convection in this context has nothing to do with turbulent convertion, but refers only to the flow of mass, momentum, and energy past a point in space as a result of the fluid motion. Performing the integrations over the cell volumes symbolically, we difference Eq. (11) for the internal energy in Lagrangian form as

$M_{c, j}{ }^{n+1} I_{j}{ }^{n+1}-M_{c, j}{ }^{n} I_{j}^{n}=\delta t R_{j}{ }^{n+1} v_{c, j}{ }^{n}$

or equivaleritly

$$
I_{j}{ }^{n+1}=\frac{M_{c, j}{ }^{n}}{M_{c, j}{ }^{n+1}}\left(I_{j}{ }^{n}+\frac{\delta t R_{j}{ }^{n+1} v_{c, j}{ }^{n}}{M_{c, j}{ }^{n}}\right) .
$$

If there $i_{s}$ no diffusion in the mass equation, i.e., if $\sigma=0, M_{c, j}{ }^{n+1}=M_{c, j}{ }^{n}$ for a Lagrangian calculation. towever, this is not true in general, and care must be taken to include the mass factor properly. Our procedure is to is ilude it in the rezone phase so that $M_{c, j}{ }^{n+l}$ contains contributions from both the turbulent diffusion and the convection. This means that the effect of $a$ on $a$ is not included fully implicitly in the I equation, althuugh it may be partially done through the dependence of $R$ on $P$. Unless the effect of turbule "mass diffus.on in one time step is large this should not lead to stability problems.

The calculational mesh is labeled with half integral indices at the vertices as indicated in Fig. 1 . The quantities $F, P_{H}, I, P$ and $M_{C}$ are cell-centered quantities, labeled wi th integral values; coordinates, velocities and vertex masses are assigned at vertices. The time level is indicated with a

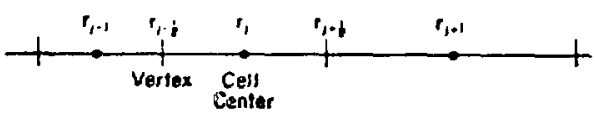

Cell centered quentities, $P, \rho_{1} \rho_{H}, I_{1}, \mu_{,} M_{C, *}$

vertex quantities' $u, K, M$,

Fig. 1. A segment of the VEGA computing mesh that illustrates the subscript convention.

superscript.

In order to set up a system which is linear in the time advanced quantities, it is necessary to expand the nonlinear terms about the old time leval, as for example

$(\omega I)^{n+1}=\rho^{n} I^{n+1}+\rho^{n+1} I^{n}-\rho^{n} I^{n}$.

This ignores the quadratic terms in the changes of the variables; in Eq. (13) we have dropped the term $\left(n^{n+1}-p^{n}\right)\left(I^{n+1}-I^{n}\right)$. Consequentiy, the equations are not fully time advancer and, should the quadratic tem be appreciable compared to . " 1 , numerical difficulties can occur.

As far as $R_{j}{ }^{n+1}$ is concerned, this can be a nonlinear function of the variables. Monetheless, we attempt to include as much time-advanced information as possible in its evaluation. Consider that part of $R_{j}$ that arises from the energy released in nuslear reactions. This is, in a single calculational cycle,

$\delta(D I) \sim D E \delta t \quad$

Because $c$ is, in general, a complicated function of $D, D_{H}$, and $d$, one might most simply include it explicitly. This could seriousiy limit the time step. In fact $t$ increases more rapidly than linearly with temperature which allows us to include some advanced time information by factoring out the internai energy I. Rates for the carbon cycle and for the proton chain are proportional to the first and second powers of $\mathrm{B}_{H}$, respectively. For this reason we factor out $\rho_{H}$ also and write

$\varepsilon\left(\rho, \rho_{H}, I\right)=\varepsilon_{0}\left(\rho, \mu_{H}, I\right) \rho_{H} J$, 
then linearizing the product, " $I$, in the advanced time quantities. Although this tern cannot be made fully implicit in the direct solution approach, the present treatment greatly improves stability over the explicit method.

Note that instead of using an equation for the total energy, we elect to calculate the specific internal energy. Experience has shown that the use of this equation is more accurate than the use of the total energy equation because errors in the kinetic energy are not then buried in the internal energy. Since the pressure depends on the temperature, as do some of the more crucial physical parameters, it is important to obtain the internal energy as accurately as possible. What we sacrifice is total energy conservation.

The primary stability limit of our method is

$u \frac{d t}{\delta x}<1$

In practice we limit $u d t / \approx x$ to somewhat less than 0.5 over the entire mesh. If a quadratic term in the change of any two variables is large with such a time step, we cannot expect unconditional stability. For this reason we include an additional limit on the time step such that if any variable changes fractionally by more than a fixed amount in any cell, the time step is decreased. This prevents instabilities that might occur because of the partially explicit rature of the solution. With this test the quadratic term in the variable changes is effectively limited. The optimum value for the fractional change will clearly be problem dependent.

We now illustrate the differencing of the equation for a vertex quantity with the Lagrangian form of the monentum equation:

$\frac{d}{d t} \iiint_{v} \rho u d^{3} v=\iiint_{v}\left(-\rho g-\frac{\partial p}{\partial r}+\Lambda\right) d^{3} v$.

Since the velocity is defined at vertices, the control volume over which the integration is performed runs from cell center to coll center, that is from $r_{j}$ to $r_{j+l}$. Defining a vertex mass as the average mass of the adjacent cells,
$\iiint_{V} d^{3} V=M_{v, j+\frac{1}{2}}=\frac{1}{2}\left(M_{c, j}+M_{c, j+1}\right)$,

we difference Eq. (17) in a straightforward fashion to arrive at Eq. $(\mathrm{A}-28)$ in the Appendix. For completeness the Appendix contains the difference equations for the renaining variables and includes a discussion of some of the considerations which entered into decisions concerring the manner of differencing.

It must be remembered that it is necessary to couch the equation of state in such a fashion that it too can be linearized. Otherwise, point relaxation techniques would have to be employed.

\section{GAS PHYYSICS}

Because in this initial development our emphasis has been on the solution algorithm for the basic fluid equations rather than on the details of the gas physics, we have limited the constitutive relations to fairly simple form. At the same time, we have included sufficient generality to solve a broad class of problems.

We assume the gas consists of three nuclear species: hydrogen, helium, and metals (atomic number $>2$ ) with a fixed mass fraction $z$. The equation of state allows for hydrogen to be converted to helium, allows for partial ionization and for $\mathrm{H}_{2}$ dissociation, and includes radiation pressure and nonrelativistic electron degeneracy.

A. Equation of State

Uncier the assumptions listed above, the total pressure is

$$
\begin{aligned}
P & =\frac{1}{3} a T^{4}+\frac{k T}{H}\left[\rho_{H}\left\{\frac{1}{H_{H}}-\frac{1}{\mu_{H e}}+\Psi\left[\frac{n_{H}}{\mu_{H}}-\frac{n_{H e}}{\mu_{H e}}\right]\right\}\right. \\
& \left.+p\left\{\frac{(1-z)}{{ }^{H} H e}+\frac{2}{\omega_{2}}+\because\left[\frac{(1-2) n_{H e}}{\omega_{H e}}+\frac{2 n_{Z}}{\mu_{2}}\right]\right\}\right] .
\end{aligned}
$$

In the above, $a$ is the Stefan-Boltzmann constant, $k$ is the Boltzmann constant, $H$ is the mass of one atomic mass unit, $\omega_{i}$ is the average molecular weight of the nuclei of species $i, n_{i}$ is the average number of free electrons per nucleus of species $i$. The degeneracy is included through the ratio 
$:=\frac{2}{3} \frac{F_{3 / 2}(n)}{F_{1 / 2}(n)}$

where $F_{n}(\cdot)$ is the Fermi-birac integral of order $n$,

$F_{n}(\cdot)=\int_{0}^{\infty} \frac{:^{n} d t}{1+\exp (t-n)}$

and $:$ is the degeneracy parameter. We have made use of the relation

$\therefore \mathrm{He}=(1-2)+-\mathrm{H}$

to eliminate: $\mathrm{He}$. The degeneracy factor : is evaluated from the expressions given by Larson and Demarque. ${ }^{5}$ and $n_{j}$ and.$_{j}$ are evaluated using the ionization-dissociation equation of state from the stellar envelope prcgram of Paczynski. 6

\section{B. Specific Heat}

The internal energy per gram is

$I=c_{v} T=\frac{a T^{4}}{g}+I_{e x}$

$$
\begin{aligned}
& +1 . \frac{5 k T}{H}\left\{\frac{\rho_{H}}{i}\left[\frac{1}{i_{H}}-\frac{1}{n_{H e}}+:\left(\frac{n_{H}}{i_{H}}-\frac{n_{H e}}{{ }^{{ }^{H}}}\right)\right]\right. \\
& \left.+\left\{\frac{(1-Z)}{{ }_{H e}}+\frac{z}{z_{z}}+:\left(\frac{(1-Z) n_{\mathrm{He}}}{u_{\mathrm{He}}}+\frac{Z n_{z}}{u_{z}}\right)\right]\right\} .
\end{aligned}
$$

where $I_{\text {ex }}$ includes the specific ionization energy, the $\mathrm{H}_{2}$ dissociation eneray and the $\mathrm{H}_{2}$ vibrational, rotational, and excitation energies. We define lex so that it vanishes at zero temperature. With this defirition of $I_{e x}, c_{v}$ is the temperature averaged value of the thermodynamic derivative (JI/JT) with density art composition fixed.

C. Nuclear Reaction Rate

We use the nuclear reaction rates of Larson and Demarque for the proton chain and carbon cycle. These rates assume equilibrium abundances, but the three branches of the proton chain are followeo separately. The nuclear energy production rate $\varepsilon$ is a complicated nonlinear function of $\rho, T$, and composi- tion, and it will not be reproduced here.

D. Viscosity

Two regimes are of interest in estimating the viscosity of the stellar gas. At low temperatures the dominant source of viscosity is collisions between atoms. As a crude estimate for this regime, we can fit Edmonds ${ }^{7}$ viscosity table with the form

$\mu=7.15 \times 10^{-6} \mathrm{~T}$.

Interactions betveen atims and photons dominate the viscosity at higher tremperatures. ${ }^{8}$ so that

$\therefore=\frac{4 a}{15 c} \frac{T^{3}}{k s}$

where $k$ is the Rosseland mean opacity.

E. Consuctivity

In radiative zones, we use

$k_{\text {rad }}=-\frac{d}{3} \cdot \frac{T^{3}}{k f}$

The: opacity tables used for $T \cdot 2000^{\prime} \mathrm{l}$ were taken from Paczynski's stellar envalone program. These tables are cox-Stewert tab?es ${ }^{9}$ with an appraximate correction for water vapor opacity. At temperatures below $1500^{\circ} \mathrm{K}$, grains dominate the opacity. and the constant value, $=0.15$, used by Larson 10 was chosen. Recent work by Alexander 11 indicates that this value is perhaps a inttor of six too low. Between $1500^{\circ} \mathrm{K}$ and $2000^{\prime} \mathrm{K}$, a linear interpolation between the two regimes is used.

F. Turbulence Modeling

At this point in the development, we have not incorporated complete turbulence model. However, we have included turbulent diffusion of mass and heat. The Schwarzschild criterion is used to detuct regions of turbulent convection. If we define

$$
\frac{\Gamma_{2}}{\Gamma_{2}-1}=\frac{12 \beta(1-\beta)\left(\gamma_{g}-1\right)+\beta^{2}+(4-3 \beta)^{2}\left(\gamma_{g}-1\right)}{(4-3 \beta)\left(\gamma_{g}-1\right)},
$$


where $Y_{g}$ is the ratio of specific heats for the gas alone, and $i$ is the ratio of gas pressure to total pressure, then in unstable zlines the effective thermal diffusion coefficient for adiabatic convection is

$k_{\text {con }}=\frac{\ddot{2}}{2-T} \frac{F}{g !}$.

$F$ is the energy flux and $g$ is the local gravitational acceleration. Analogou's expressions cali easily be derived for other theories of turbulent convection, but this expression is adequate for our preliminary calculations.

The turbulent diffusion of mass is discussed by Cloutman ${ }^{2}$ and by Rivard, Butler, and Farmer. ${ }^{3}$ We use equal values for the turbulent diffusivities of mass and internal energy (i.e., the turbuient Schmidt and turbulent Prandt I numbers are assumed to be equal). Thus, in convection zones,

$\therefore=\frac{x_{\operatorname{con}}}{a_{v}} ;$

: yarishes in radiative zones.

A more reliable approach to the treatment of convection zones would be a turbulence trinsport model along the iines of Daly and Harlow, ${ }^{12}$ or Rivard, et al. Another usable, but less reliable, model could be based on the mixing length theory.
Further investigation of turbulence modeling for astrophysical applications is in progress.

\section{SOLVING THE LINEAR SYSTEM}

One time step is accomplished in two distinct phases. In Phase I, we do an implicit Lagrangian time step; in Phase 11 , we do the rezuning. After rezoning we do any necessary bookkeeping and output. In this section, we discuss some computational details for carrying out these phases.

In Phase 1, the lirizar difference equations we want to solve are Eqs. $(A-3),(A-18),(A-24),(A-26)$, $(A-27)$ and a species equation similar to $(A-18)$ that includes nuclear burning. Thus we have $6 \mathrm{~N}$ equations if there are $N$ real cells. However, the matrix is sparse and block diagonal, with one 6 by 18 block for each cell as shown schematically in Fig. 2 . This structure is similar to the structure of the inatrix which occurs in the Henyey method. However, ve sulve our linear system only once per time step. One would expect, thisn, that our code uses about as much time for one time step as the Henyey method nueds for one iteration. Cumparison with Henyey method programs by Paczjnski and by Larson and Demarque verify this observation.

The order of the six equations within each block and the order of the variables in the solution vector is very important for efficient matrix inverision.

\section{Voriables}

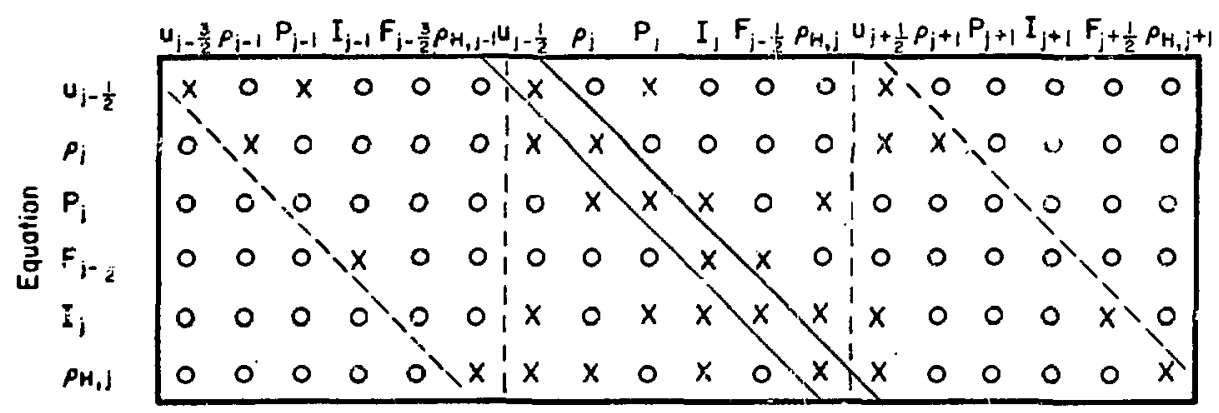

Fig. 2. A representative portion of the banded matrix that is inverted to solve the fluid equations for cell 1. Nonzero matrix elements are marked with $x^{\prime} s$. The symbols at the top show the order of the variables in each equation; the symbols at the left indicate which equation is associated with a given row. 
Rather than use a block diagonal routine, we used a banded system solver that uses LU decomposition with partial pivoting. 13 The diagonal is inside the solid lines, and the nonzero matrix elenents are confined to the band indicated in Fig. 2 by dashed lines. Since the running time of this routine is proportional to the cube of the bandwidth, and most of our running time is spent in the matrix routines, it is crucial to minimize the bandwidth. The layout shown in Fig. 2 is optimun, with a bandwidth of 13 . If a bandwidth of 14 were used, the code would run about 25: slower. A layout witil nonzero elements in the upper right and lower left corners of each block would have a bandwidth of 23 and lead to a code that would be about 5.5 times sliwer. The narrow bandwidth also minimizes memory requirements and rounding errors. Running time and storage requirements would be prohibitive if one attempted to store and operate on the full matrix. The solution vector is given by the variables in the order shown across the top of the block in Fig. 2.

Eoundiry conditions are impesed by writing special difference equations for the innermost and outermost real cells. The inner boundary conditions at the origin are

$u(0, t)=F(0, t)=0$.

where $F$ is the seat flux, and

$\left.\frac{y_{i}}{3 r}\right|_{r=0}=0$

$\left.\frac{\partial I}{\partial r}\right|_{r=0}=0$

It is not necessary to specify the pressure in the inner fictitious cell.

The outer boundary conditions present a challenging problem that does not have a unique solution. For the protostar problem, we use a rigid wall:

$u_{N-3 / 2}=0$,

$\left.\frac{\partial \theta}{\partial r}\right|_{r=R_{0}}=0$, \begin{tabular}{l:l}
$H$ & \\
\hdashline$r=R_{0}$ & $=0$.
\end{tabular}

The temperature is a fixed constant on the boundary. $r=R_{0}$. In the above, $N-$. is the index of the outer boundary. The boundary temperature for the protostar problein is $10 \mathrm{~K}$. ine nomal equations for $p$ and $F$ are used on the outer boundary.

The detailed equations used in Phase 1. are given in the Appendix but the philcsophy of Phase Il will be described here. We assume we have completed the Lagrangian time step of Phase I and that we have specified the velocity $u_{q, j+-}$. with which we want to move each grid point. In our schemp. the grid foints cannot move nore tian a cell width or instability results. We compute the fluxes of mass, momentum, and eneryy through the moving vertices and cell centers, and use these fluxes to compute the amount of each conserved quantity that passes through each moving surface as it moves from its original position to its final position. Then this amount is transferred from one side of the surface to the other, thereby conserving mass, momentum, and energy to within computer rounding errors. From the original amount of mass, momentum, and energy in each cell and the changes due to rezoning, we can then recover the densities, velocities, and intermil energies in each cell after rezoning. Finally we do bockkeeping, compute $p_{j}{ }^{n+1}$ from the equation of state, generate output (if any) and begin the next time step.

\section{v. SAFPILE CALCULATION}

As an example of a calculation performed with VEGA, we present the early phases of collapse of a one-solar-mass protostar. We have tried to reproduce the results of Larson, 10 and we find good agreement among the results from VEGA, Larson, and a special ICE method program. Larson used a variation of the so-called Henyey method 5,14 and was limited to about 50 mesh cells in his calculations. The special ICE code is a one-dimensional, spherical coordinate variation of the basic ICE method. 15 This variation includes a nonuniform mesh and an implicit internal energy equation.

The initial condition for our test problem is a one-solar-mass, uniform cloud of gas at $10^{\circ} \mathrm{K}$. 
The iydrogen is assumed to be in the foril of $\mathrm{H}_{2}$ and the adius of the cloud is

$\mathrm{Pe}_{\mathrm{C}} \quad 0.41 \frac{\mathrm{GM}}{\mathrm{RT}}=1.633 \times 10^{17} \mathrm{~cm}=$

where $R$ is the universal gas coristant divided by the mean holecular weight. This cloud is just unstable toward gravitational collayse accurding to the Jeans criterion. We assume the gas is a verfect gamia-lan qus with: 1.4 , and the specific heat, $c_{y}$. and mean molecular weight are assimed to be constants. We neglect changes of excitation eneray in the $\mathrm{H}_{2}$ molecules and tarminate the calculation befora discociasion becomes important. We neglect all anisotropic phenomend sach as magnetic fiolds and rotation.

The code was run in an Eulerian mode with 150 cells. The radius of the central cel! was $5,1 n^{12}$ cill the cell wideh growe as, function of radius by a constant factor to crive; tric entire radius with 150 cells. The outer houndary cundition is a fixed. Figid wall (u = n) at $10 \%$.

rigure 3 shows the density and velocity profiles at several phases of collapse. The left-hand panel covers the entire radius of the cioud, while the right-hand panel covers only the innemost 4.1 of the radius. The curve labeled, $(0)$ is the initial density, and the initial velocity is zero.

The curves labeled. (300) and $u(300)$ are the ciensity and velocity, respecively, after 300 cycles. At this stage, the fluid is sill isothemal at $10^{\circ} \mathrm{K}$.

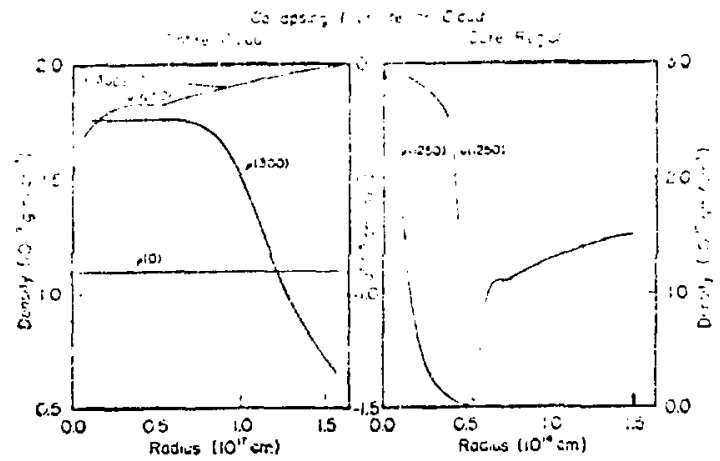

Fig. 3. The density and velocity profiles of a onesolar mass protostar at several different times. The numbers in parentheses denote the cycle numbers.
A rarefaction wave is moving inward at the local sound speed relative to the collapsing fluid. The velocity is linear and subsonic on both sides of the rarefaction. which is near $r=1.0 \times 10^{37} \mathrm{~cm}$. Inside the rarefoction, the density is spatially carstant but increases with time. Outside the rarefaction, the density drops as $1 / r^{2}$. This behavior is expected theoretically.

At 1250 cycles the entire character of the soistion has changed. The rarefaction has hit the center and a nearly static core with a radius of $5 \times 10^{13}$ cn has fomed. Larson reports a core radius of $5 \times 10^{13} \mathrm{~cm}$. In the outer half of the cloud, the velocity has hardly changed since cycle 300 . However, the infalling material goes supersonic outside the core. The velocity ininimum at $5 \times 10^{13}$ is abou: Much 7. An accretion shock surrounding the cone rapidly drops the velocity to Mach 1 at the surface of the core, and the velocity drops roughlv linearly to zero at the origin. The core is now ontically thick, and the center temperature is about $170 \mathrm{k}$

The velocity profile just outside the accretion shock shows an appreciable oscillation. This is a numerical effect due to dispersive truncation errors. The true shock Mach number is probably rloser to five than to seven. This problem was run with no viscosity. He could help smooth our solution by increasing the donor cell parameter, which has the same effect as artificial viscosity because it increases the diffusional truncation errors. In fact. it is the combinatich of an Eulerian calculation with iarge donor cell that allows us to run the strong shock stably withuut explicitly adding an artificial viscosity.

Figure 4 shows the central density as a function of time as computed by three different methods. The agreenen: among the three methods is impressive until shortly after $5 \times 10^{12}$ secs, which is shortly before the rarefaction hits the origin. What happens mext is a complicated series of radial pulsations. The details of these pulsations are sensitive to the numerical technique used to integrate the equations. Larson ${ }^{16}$ reports that the pulsations occurred with his Lagrangian calculations, but not with nis Eulerian calculations. Because Eulerian methods tend to be more diffusive than Lagrangian methods, it appears that the pulsations 


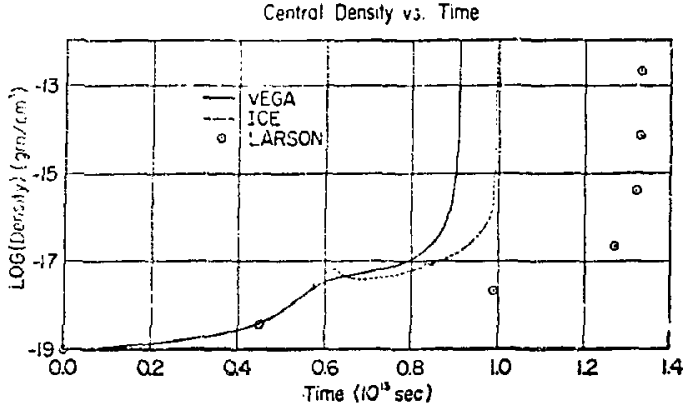

Fig. 4. Central density of a one-solar-mass protostar as computed $y$ three different programs.

are weakly ariven at first and then easily damped. These pulsations temporarily halt the collapse in the core. The longer the core pulsates, the longer the rapia density rise is delayed. We feel that the difserences in the time of onset of the collapse are due to the $n$ irical differences in the various techniques during the pulsation phase. It is difficult to assess the numerical effects of Larson's methods. The ICE code employs a special truncation error cancellation scheme 17 to reduce numerical diffusion appreciably compared to the standard ICE method. The density bump at $6 \times 10^{12}$ secs is due to the first expansion after the rarefaction reaches the origin. Several ascillations follow, but their effect is too small to show on the figure except at $8.5 \times 10^{12} \mathrm{sec}$. The VEGA calculation is more diffusive, resulting in lower amplitude and faster damping in the pulsations. While the flow velocity in the core actually changes sign in the ICE calculation, it does not in the VEGA calculation. The VEGA collapse merely goes in spurts.

APPENDIX

THE DIFFERENCE EQUATIONS

I. EQUATION OF STATE

We include in the code the general equation of state

$P=\Gamma a !+\frac{1}{3} a T^{4}$

where $\Gamma$ is a function that can represent all real gas effects. The special case,

$P=(\gamma-1) \rho I+\frac{1}{3} a\left(\frac{I}{c_{v}}\right)^{4}$,

which of ten suffices, is approximated by

$$
\begin{aligned}
p_{j}^{n+i} & -\left[(\gamma-1) I_{j}^{n}\right] \rho_{j}^{n+1} \\
& -\left[(\gamma-1) \rho_{j}^{n}+\frac{4 a}{3 c_{y, j} n}\left(T_{j}^{n}\right)^{3}\right] I_{j}^{n+1}
\end{aligned}
$$

$$
=-P_{j}^{n}-\frac{2 a}{3}\left(T_{j}^{n}\right)^{4} \text {. }
$$

Empiricaliy, we find this form to be unconditionally stable with respect to the usual courant condition. Equation $(A-3)$ was used without radiation pressure in the protostar calculation.

The best treatment of equations of state with variable $\Gamma$ is unknown; we are continuing our effort to clarify this point. A natural extension of $\mathrm{Eq}$. $(A-3)$ is to replace $(\gamma-1)$ by $\Gamma_{j}{ }^{n}$. It is not clear at this point if this procedure is unconditionally stable. There is some indication that it may be necessary to use a more implicit scheme such as

$$
\begin{aligned}
P_{j}^{n+1} & -\left[\rho_{j}^{n}\left(\Gamma_{j}^{n}+I_{j}^{n}\left(\frac{\partial \Gamma}{\partial T}\right)_{j}^{n}\right)+\frac{4 a}{3 c_{v, j} n}\left(T_{j}{ }^{n}\right)^{3}\right]_{j}^{n+1} \\
& -\left[I_{j}^{n}\left(r_{j}^{n}+\rho_{j}^{n}\left(\frac{\partial \Gamma}{\partial \rho}\right)_{j}^{n}\right)\right]_{j}^{n+1}
\end{aligned}
$$




$$
\begin{aligned}
& =-p_{j}{ }^{n}-\frac{2 a\left(T_{j}^{n}\right)^{4}}{3}-\rho_{j}{ }^{n} I_{j}{ }^{n}\left[\rho_{j}{ }^{n}\left(\frac{\partial \Gamma}{\partial \rho}\right)_{j}^{n}\right. \\
& \left.+I_{j}{ }^{n}\left(\frac{\partial \Gamma}{\partial I}\right)_{j}^{n}\right] .
\end{aligned}
$$

This scheme has not been tested, but we expect it to have good stability properties; noreover, it provides a method for using equation of state tables that contain $\Gamma$ and perhaps its derivatives.

For certain special equations of state, it may be possible to use explicit mean molecular weights and write the linearized equation of state in termis of $p_{j}, p_{H_{3} j}$, and $I_{j}$. For example, E4. (19) may be approximated by

$$
\begin{aligned}
P_{j}{ }^{n+1} & -\frac{I_{j}{ }^{n+1}}{c_{v, j}{ }^{n}}\left\{\frac{4 a\left(T_{j}{ }^{n}\right)^{3}}{3}+\frac{k}{H}\left[\rho_{H, j}{ }^{n} B_{H, j}+\rho_{j}{ }^{n} B_{H e, j}\right]\right\} \\
& -\rho_{H, j}{ }^{n+1}\left\{\frac{k T_{j}^{n}}{H} B_{H, j}\right\}-\rho_{j}{ }^{n+1}\left|\frac{k T_{j}^{n}}{H} B_{H e, j}\right| \\
& =-p_{j}{ }^{n}-\frac{2 a}{3}\left(T_{j}{ }^{n}\right)^{4}
\end{aligned}
$$

where

$\xi_{H, j}=\left[\frac{1}{\mu_{H}}-\frac{1}{\mu_{H e}}+\psi\left(\frac{n_{H}}{\mu_{H}}-\frac{n_{H e}}{\mu_{H e}}\right)\right]$

and

$B_{\mathrm{He}, \mathrm{j}}=\left[\frac{(1-7)}{\mu_{\mathrm{He}}}+\frac{z}{\mu_{z}}+\psi\left(\frac{(1-Z) n_{\mathrm{He}}}{\mu_{\mathrm{He}}}+\frac{Z n_{z}}{\mu_{z}}\right)\right] \cdot(\mathrm{A}-7)$

We have used Larson and Demarque's 5 approximation to $\psi$ :

$\Psi=\left(1+0.1938 F_{\frac{1}{2}}(n)\right)^{5 / 3} /\left(1+0.12398 F_{\frac{1}{2}}(n)\right)$, where

$$
F_{\frac{1}{2}}(n)=\left[\frac{h^{3}}{4 \pi H\left(2 m_{e} k\right)^{3 / 2}}\right] \frac{\rho}{\mu_{e} T^{3 / 2}}
$$

and $\mu_{e}$ is the mean molecular weight per free electron; $h$ is Planck's constant. The stability properties of this form are not clear; it may be necessary to make the function $\Psi$ partially implicit with an approximation such as

$$
\begin{aligned}
\psi_{j}^{n+1} \approx \psi_{j}^{n} & +\left(\frac{\partial \Psi}{\partial \rho}\right)_{j}^{n}\left(\rho_{j}^{n+1}-\rho_{j}^{n}\right) \\
& +\left(\frac{\partial \psi}{\partial I}\right)_{j}^{n}\left(I_{j}^{n+1}-I_{j}{ }^{n}\right)
\end{aligned}
$$

\section{CONTINUITY EQUATION}

Let us write the continuity equation as

$\frac{d \rho}{d t}+\rho D=\frac{1}{r^{2}} \frac{\partial}{\partial r}\left(r^{2} \sigma \frac{\partial \rho}{\partial r}\right)$

where

$D=\frac{1}{r^{2}} \frac{\partial}{\partial r}\left(r^{2} u\right)$.

Define the quantity

$d_{j}^{n}=-\frac{\delta t}{1+\delta t D_{j}^{n}}$

In spherical coordinates,

$D_{j}{ }^{n}=\frac{u_{j+\frac{1}{2}}^{n}-u_{j-\frac{1}{2}}{ }^{n}}{r_{j+1 / 2}-r_{j-1 / 2}}+2 \frac{u_{j+\frac{1}{2}}{ }^{n}+u_{j-1 / 2} n}{r_{j+\frac{1}{2}}+r_{j-1 / 2}}$.

Then Eq. $(A-11)$ is approximated by

$$
\rho_{j}{ }^{n+1}-\sigma_{j}{ }^{n} \rho_{j}{ }^{n} D_{j}{ }^{n+1}+\lambda_{j}{ }^{n}\left[\frac{1}{r^{2}} \frac{\partial}{\partial r}\left(r^{2} \sigma^{n} \frac{\partial \rho^{n+1}}{\partial r}\right)\right]_{j}
$$

$$
=\rho_{j}^{n} \text {. }
$$


Defining normalized cell volumes

$V_{c, j} \equiv r_{j+3_{i}}^{3}-r_{j-i_{i}}^{3}$

and normalized vertex areas

$A_{j-\frac{1}{2}}=3 r_{j-1 / \frac{1}{2}}^{2}$

we approximate Eq. (A-11) by

$$
\begin{aligned}
& \rho_{j}^{n+1}\left\{1-\frac{2 d_{j}^{n}}{V_{c, j}}\left[\frac{\sigma_{j+\frac{1}{2}}{ }^{n} A_{j+\frac{1}{2}}}{r_{j+3 / 2}-r_{j-\frac{1}{2}}}+\frac{\sigma_{j-\frac{1}{2}}{ }^{n} A_{j-\frac{1}{2}}}{r_{j+\frac{1}{2}}-r_{j-3 / 2}}\right]\right\} \\
& +\rho_{j+1}^{n+1}\left\{\frac{2 d_{j}^{n} \sigma_{j+\frac{3}{2}}^{n} A_{j+\frac{1}{2}}}{v_{c, j}\left(r_{j+3 / 2}-r_{j-\frac{1}{2}}\right)} \mid\right. \\
& +\rho_{j-1}{ }^{n+1}\left|\frac{2 d_{j}{ }^{n} \sigma_{j-\frac{1}{2}}{ }^{n} A_{j-\frac{1}{3}}}{V_{c, j}\left(r_{j+\frac{1}{2}}-r_{j-3 / 2}\right)}\right| \\
& +u_{j+\frac{1}{2}}^{n+1}\left[-d_{j}^{n}\left(\frac{2}{r_{j+\frac{1}{2}}+r_{j-\frac{1}{2}}}+\frac{1}{r_{j+\frac{1}{2}}-r_{j-\frac{1}{2}}}\right)\right] \\
& +u_{j-\frac{1}{2}}^{n+1}\left[-d_{j}^{n}\left(\frac{2}{r_{j+\frac{1}{2}}+r_{j-\frac{1}{2}}}-\frac{1}{r_{j+\frac{1}{2}}-r_{j-\frac{1}{2}}}\right)\right] \\
& =\rho_{j}{ }^{n} \text {. }
\end{aligned}
$$

As with all our difference equations, the geometric quantities, $r, A$, and $V$ are always the old-time values. The left-hand side of Eq. (A-11) is differenced directly because the integration procedure would frovide no immediate infonmation on the new densities. It would merely give us the trivial fact that the mass in a cell does not change in the absence of turbulence. The advanced time-cell volume, necessary to compute the advanced time density from the cell mass, is not directly available. Only the diffusion tem in differenced as if we followed the integration procedure described in Sec. II for the momentuin and energy equations.
III. SPECIES EQUATION

The species equation is identical to the continuity equation except for the nuclear term. The nuclear term is written as

$$
-d_{j}^{n}\left(\frac{\delta \rho_{H}}{\delta t}\right)_{j}=-d_{j}^{n} \rho_{j}^{i !}\left(\frac{m_{H} n}{Q}\right) t_{o j}\left(\rho_{H, j}{ }^{n+1} 1_{j}^{n}\right.
$$

$$
\left.+\rho_{H, j}{ }^{n} I_{j}{ }^{n+1}-\rho_{H, j}{ }^{n} I_{j}{ }^{n}\right),
$$

where $\pi_{H}$ is the mass of one atom of species $H, n$ is the number of $\mathrm{H}$ atomis destroyed per reaction that releases energy $Q$, and

$\varepsilon_{0 j}=\frac{\varepsilon_{j}^{n}}{\rho_{H, j}^{n} I_{j}^{n}}$.

The term $(A-19)$ is added to the right-hand side of Eq. $(A-18)$, and, of course, $\rho_{H}$ replaces $\rho$.

IV. ENERGY EQUATION

The equation for the specific internal energy may be written as

$\frac{\partial \rho I}{\partial t}+\frac{1}{r^{2}} \frac{\partial}{\partial r}\left(r^{2} \rho \mathrm{I}\right)=-p D+\frac{1}{r^{2}} \frac{\partial}{\partial r}\left(r^{2} K \frac{\partial T}{\partial r}\right)+\rho \varepsilon+\Phi$.

For numerical reasons we have found it expedient to define the flux

$F=k \frac{\partial T}{\partial r}=k \frac{\partial}{\partial r}\left(\frac{I}{c_{V}}\right)$

and carry along this extra equation. In both the above $K=\operatorname{Max}\left(K_{\text {rad }}, K_{c o n}\right)$. The diffusion term in Eq. $(A-21)$ is then calculated as

$\frac{1}{r^{2}} \frac{\partial}{\partial r}\left(r^{2} F\right)$

This is necessary whenever $x \equiv \log _{10}\left(k \delta \mathrm{t} / \rho c_{v} \delta r^{2}\right)$ approaches or exceeds the number of digits carried in the calculation, because the coefficient matrix 
has a term $1 j$ ke $1+2 \times 10^{x}$. The one gets lost in round off if $x$ is too iurge, and the matrix package cannot successfully recover the one in the course of solving the linear system.

In Eq. (A-21) we define the variable, $\Phi$, for the viscous work, that is,

$\Phi \equiv \mu\left[\left(\frac{\partial u}{\partial r}\right)^{2}+\frac{2 u^{2}}{r^{2}}\right]+\lambda\left[\frac{1}{r^{2}} \frac{\partial}{\partial r}\left(r^{2} u\right)\right]^{2}$.

The difference approximation to Eq. $(A-21)$ is derived by integrating over the spherical shell between $r=r_{j-1_{5}}$ and $r=r_{j+t_{2}}$, using the procedure described in Sec. 11. The compressional work term is time rentered to conserve energy; everything else is made as implicit as possible consistent with our constraint of linear equations.

$$
\begin{aligned}
& I_{j}{ }^{n+1}=I_{j}{ }^{n}+\delta t\left\{\varepsilon _ { o j } \left[o_{H, j}{ }^{n+1} i_{j}{ }^{n}+o_{H, j}{ }^{n} I_{j}{ }^{n+1}\right.\right. \\
& \left.-\rho_{H, j}{ }^{n} I_{j}{ }^{n}\right]+\frac{V_{C, j}}{M_{c, j}} \Phi_{j}+\frac{1}{M_{c, j}}\left[A_{j+\frac{1}{2}} F_{j+\frac{2}{2}}{ }^{n+1}\right. \\
& \left.-A_{j-\frac{1}{2}} F_{j-\frac{1}{2}}^{n+1}\right]-\frac{1}{2 M_{c, j}}\left[p _ { j } ^ { n + 1 } \left(A_{j+\frac{1}{2}} u_{j+\frac{1}{2}}^{n}\right.\right. \\
& \left.-A_{j-\frac{1}{2}} u_{j-\frac{1}{2}}^{n}\right)+p_{j}^{n}\left(A_{j+\frac{1}{2}} u_{j+\frac{1}{2}}^{n+1}\right. \\
& \left.\left.-A_{j-\frac{1}{2}} u_{j-1}^{n+1}\right)\right] \text {, }
\end{aligned}
$$

where

$$
M_{c, j}{ }^{n}=\rho_{j}{ }^{n} v_{c, j},
$$

and

$$
\begin{aligned}
\Phi_{j} & =\mu_{j} n \mid\left[2\left(u_{j+\frac{1}{2}}^{n+1}-u_{j-\frac{1}{2}}^{n+1}\right)\left(u_{j+\frac{1}{2}}^{n}-u_{j-\frac{1}{2}}{ }^{n}\right)\right. \\
& \left.-\left(u_{j+\frac{1}{2}}^{n}-u_{j-\frac{1}{2}}\right)^{2}\right]\left(r_{j+\frac{1}{2}}-r_{j-\frac{1}{2}}\right)^{-2} \\
& +\left[4\left(u_{j+\frac{1}{2}} n+1+u_{j-\frac{1}{2}} n+1\right)\left(u_{j+\frac{1}{2}} n+u_{j-\frac{1}{2}} n\right)\right.
\end{aligned}
$$

$$
\begin{aligned}
& \left.-2\left(u_{j+l_{2}}{ }^{n}+u_{j-l_{i}}\right)^{2}\right]\left(r_{j+l_{i}}+r_{j-\frac{1}{j}}\right)^{-2} \\
& -\frac{2}{3\left(v_{c, j}\right)^{2}}\left[2\left(A_{j+\frac{1}{2}} u_{j+\frac{1}{2}} n+1-A_{j-\frac{1}{2}} u_{j-\frac{1}{2}}{ }^{n+1}\right) x\right.
\end{aligned}
$$$$
\left(A_{j+\frac{1}{2}} u_{j+\frac{1}{2}}^{n}-A_{j-\frac{1}{2}} u_{j-\frac{1}{2}}^{n}\right)
$$$$
\left.\left.-\left(A_{j+H_{1}} u_{j+1 / 2}^{n}-A_{j-\frac{1}{2}} u_{j-\frac{1}{2}}\right)^{2}\right]\right) \text {. }
$$

Here we have assumed $\lambda=-\frac{2}{3} \mu$. The viscous dissipation term is positive-definite if all velocities are at the same time level, but we lose this physical characteristic by using velocities from a mixture of time levels. This is probably not serious, but it should be kept in mind.

The flux equation is

$$
F_{j+1_{2}}{ }^{n+1}=\frac{2 K_{j+!_{2}}{ }^{n}}{r_{j+3 / 2}-r_{j-1 / 2}}\left(\frac{I_{j+1}{ }^{n+1}}{c_{v, j+1}}-\frac{I_{j}{ }^{n+1}}{c_{v, j} n}\right) .
$$

\section{MOMENTUM EQUATION}

The momentum equation is given by

$$
\begin{aligned}
\frac{\partial \rho u}{\partial t} & +\frac{1}{r^{2}} \cdot \frac{\partial}{\partial r}\left(r^{2} \rho u^{2}\right)=-\rho g-\frac{\partial p}{\partial r} \\
& +\frac{1}{r^{2}} \frac{\partial}{\partial r}\left[r^{2}(2 u+\lambda) \frac{\partial u}{\partial r}\right]-\frac{2 u(2 \mu+\lambda)}{r^{2}}+\frac{2 u}{r} \frac{\partial \lambda}{\partial r} .
\end{aligned}
$$

The Lagrangian form of (A-27) is differenced by integrating over' a spherical shell (momentum control volume) between $r_{j-1}=\frac{1}{2}\left(r_{j-3 / 2}+r_{j-\frac{1}{2}}\right)$ and $r_{j}=\frac{1}{2}\left(r_{j+\frac{1}{2}}+r_{j-\frac{1}{2}}\right)$, using the procedure out 7 ined in Sec. II. 


$$
\begin{aligned}
& \frac{M_{v, j-\frac{1}{2}}}{s t}\left(u_{j-\frac{1}{2}}^{n+1}-u_{j-\frac{1}{2}}^{n}\right)=-M_{v, j-\frac{1}{2}}{ }^{n} g_{j-\frac{1}{2}}-2 v_{v, j-\frac{1}{2}} \frac{\left(p_{j}^{n+1}-p_{j-1}{ }^{n+1}\right)}{r_{j+\frac{1}{2}}-r_{j-3 / 2}} \\
& +\frac{4}{3}\left[A_{j} i_{j}{ }^{u_{j+1 / 2}^{n+1}}-\frac{u_{j-\frac{1}{2}}^{n+1}}{r_{j+\frac{1}{2}}}-A_{j-1 / 2} \mu_{j-1} \frac{u_{j-\frac{1}{3}}^{n+1}-u_{j-3 / 2}{ }^{n+1}}{r_{j-\frac{1}{2}}-r_{j-3 / 2}}\right] \\
& -\frac{4}{3} v_{v, j-\frac{1}{2}}\left\{\frac{u_{j-\frac{1}{2}}^{n+1}}{r_{j-\frac{1}{2}}}\left[2 \frac{\mu_{j}^{n}-\mu_{j-1}{ }^{n}}{r_{j+\frac{1}{2}}-r_{j-3 / 2}}+\frac{\left(u_{j}^{n}+\mu_{j-1}{ }^{n}\right)}{r_{j-\frac{1}{2}}}\right]\right\},
\end{aligned}
$$

where the vertex masses and vertex volumes are given by

$M_{v, j-\frac{1}{2}}=\frac{1}{2}\left(M_{c, j}+M_{c, j-1}\right)$,

$v_{v, j-1,2}=r_{j}^{3}-r_{j-1}^{3}$

The shell areas, $A_{j}$, are defined at cell centers, as indicated by the integral subscripts. To obtain the gravitational acceleration, we perform the sum

$g_{j-i}=\frac{4 \pi G}{3 r_{j-\frac{1}{2}} 2} \sum_{i=2}^{j-1} M_{c, i}$.

This procedure is more accurate for computing the gravitational potential than is solving the Poisson equation. We assume $\lambda=-2 \mu / 3$ in Eq. $(A-28)$.

\section{PHASE II}

In Phase II we are modeling the convection term

$\iint_{S} \rho Q\left(u_{y}-\tilde{u}\right) \cdot \hat{n} d S$,

where $u_{y}$ is the grid velocity and $\tilde{u}$ is the fluid velocity at the end of Phase $I$. We define the difference velocity for our one-dimensional problem, $w_{j-\frac{1}{2}} \equiv u_{g, j-\frac{1}{2}}-\tilde{u}_{j-\frac{1}{2}}$, which is the velocity of the mesh relative to tine fluid. Then $w_{j-\frac{2}{2}} A_{j-\frac{2}{2}} \delta t$ is the volume relative to the fluid that is swept out by the moving grid point. One might be tempted to take for, say the density, simply an average of the densities on either side of the moving mesh point. This is called centered differencing and is only conditionally stable. ${ }^{i 7}$ For this reason we use a mixture of centered differencing and donor cell differencing. The donor cell component adds a strong stabilizing diffusional truncation error.

Define the donor cell parameter, $\alpha_{j+\frac{1}{2}}$, by

$\alpha_{j+\frac{1}{2}}=-\bar{\alpha} \operatorname{sgn}\left(w_{j+\frac{1}{2}}\right)$,

where the function sgn is the sign of the argument, and $\bar{\alpha}$ is a consiant, $0 \leq \bar{\alpha} \leq 1$. As an example of the difference form of the convection term for a cell centered quantity,

$$
\begin{aligned}
M_{c, j}{ }^{n+1}=M_{c, j}{ }^{n} & -\frac{\delta t}{2}\left\{w _ { j - \frac { 1 } { 2 } } A _ { j - \frac { 1 } { 2 } } \left[\left(1+\alpha_{j-\frac{1}{2}}\right) \tilde{\rho}_{j-1}\right.\right. \\
& \left.+\left(1-\alpha_{j-\frac{1}{2}}\right) \tilde{\rho}_{j}\right] \\
& -w_{j+\frac{1}{2}} A_{j+\frac{1}{2}}\left[\left(1+\alpha_{j+\frac{1}{2}}\right) \tilde{\rho}_{j}\right. \\
& \left.\left.+\left(1-\alpha_{j+\frac{1}{2}}\right) \widetilde{\rho}_{j+1}\right]\right\}
\end{aligned}
$$

The tildes denote results from Phase $I$. This is a straightforward approximation to $\mathrm{Eq} .(\mathrm{A}-32)$, if $Q$ is taken to be unity. The density is obtained by calculating volumes from the new mesh position

$$
r_{j-\frac{1}{2}}^{n+1}=r_{j-1 / 2}^{n}+u_{g} \delta t .
$$


Then

$\rho_{j}^{n+1}=\frac{M_{c, j}{ }^{n+1}}{v_{c, j}{ }^{n+1}}$,

which ensures mass conservation. The convection of internal energy or mass of any species is handled in exactly the same manner.

For momentum the control volume runs from cell center to cell center, and a slight modification is necessary. The difference velocity must be obtained by averaging the difference velocities of the neighboring vertices. This leads to
4. Thompson, P. A., Compressible-Fluid Dynamics (McGraw-Hill Book Co., Inc., New York (1972).

5. Larson, R. B. and Demarque, P. R., "An Application of Henyey's Approach to the Integration of the Equations of Stellar Structure," Ap. J., 140, 524 (1964).

6. Paczynski, B., "Envelopes of Red Supergiants," Acta Astronomica, 19, 1 (1969).

7. Edmonds, F. N., "The Coefficients of Viscosity and Themal Conductivity in the Hydrogen Convection Zone," Ap. J., 125, 535 (1957).

8. Ledoux, P. and Walraven, T., "Variable Stars," Handbuch der Physik, 51, ed. S. Flugge (SpringerVerlag) (1958).

$$
\begin{gathered}
u_{j-\frac{1}{2}}^{n+1}=\frac{1}{M_{v, j-\frac{1}{2}} n+1}\left\{M_{v, j-\frac{1}{2}} n_{j-\frac{1}{2}}^{n}-\frac{\delta t}{4}\left[\tilde{\rho}_{j-1}\left(w_{j-\frac{1}{2}}+w_{j-3 / 2}\right) A_{j-1}\left(\left(1+\alpha_{j-1}\right) \tilde{u}_{j-3 / 2}+\left(1-\alpha_{j-1}\right) \tilde{u}_{j-\frac{1}{2}}\right)\right.\right. \\
\left.\left.\quad-\tilde{\rho}_{j}\left(w_{j-\frac{1}{2}}+w_{j+\frac{1}{2}}\right) A_{j}\left(\left(1+a_{j}\right) \tilde{u}_{j-\frac{1}{2}}+\left(1-\alpha_{j}\right) \tilde{u}_{j+\frac{1}{2}}\right)\right]\right\},
\end{gathered}
$$

where

$\alpha_{j}=-\bar{\alpha} \operatorname{sgn}\left(w_{j-\frac{1}{2}}+w_{j+\frac{1}{2}}\right)$.

It is not necessary to use the same $\bar{\alpha}$ in the momentum equation as in the equation for the mass or energy. We have found empirically that we need more donor cell in the mass and energy equations to keep cells from emptying out in the neighborhood of steep gradients.

\section{REFERENCES}

1. Hirt, C. W., Amsden, A. A., Cook, J. L., "An Arbi trary Lagrangian-Eulerian Computing Method for All Flow Speeds," J. Comp. Phys., 14, 227 (1974).

2. Cloutman, L. D., "Time Dependent Turbulent Mixing," Bull. Am. Ast. Soc., 7, 402 (1975).

3. Rivard, W. C., Butler, T. D., and Farmer, O. A., "The Transient Dynamics of Chenically Reactive Gaseous Mixtures with Turbulence," Proc. Fourth Int. Conf. Numerical Methods in Fluid Cynamics, ed. R. D. Richtmeyer (Springer-Verlag), 334 (1975).
9. Cox, A. N. and Stewart, J. N., "Rosseland Opacity Tables for Population I Compositions," Ap. J. Supp 1., 19, $243(1970)$.

10. Larson, R. B., "Numerical Calculations of the Dynamics of a Collapsing Proto-Star," MNRAS, 145, 27) (1969).

11. Alexander, D. R., private communication (1975).

12. Daly, B. J. and Harlow, F. H., "Transport Equations in Turbulence," Phys. of Fluids, 13, 2634 (1970).

13. Hindmarsh, A. C., Banded Linear Systems with Pivoting, UCID-30060, Lawrence Livermore Laboratory, Livermore, CA (1970).

14. Paczynski, B., "Evolution of Single Stars. I. Stellar Evolution from Main Sequence to White Dwarf or Carbon Ignition," Acta Astronomica, 20, $47(1970)$.

15. Harlow, F. H. and Amsden, A. A., "A Numerical Fluid Dynamics Calculation Method for All Flow Speeds," J. Comp. Phys. $\underline{8}, 197$ (1971).

16. Larson, R. B., Ph.D. Thesis, California Inst. of Technology (1968).

17. Rivard, W. C., Farmer, 0. A.. Butler, T. D., O'Rourke, P. J.. "A Method for Increased ACcurary in Eulerian Fluid Dynamics Calculations," Los Alamos Scientific Laboratory report LA-5426-45 (1973). 